\title{
Quedas em internamento hospitalar - causas, consequências e custos: estudo de caso numa unidade hospitalar de Lisboa
}

\author{
Ana Lúcia Romão ${ }^{a}$ Susana Nunes ${ }^{b}$ \\ ${ }^{a}$ CAPP/ISCSP, Centro de Administração e Políticas Públicas, Instituto Superior de Ciências Sociais e Políticas,

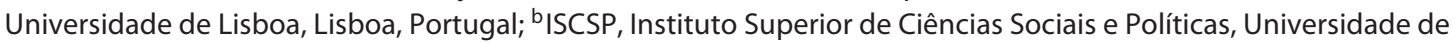 \\ Lisboa, Lisboa, Portugal
}

\section{Palavras chave}

Economia em saúde · Qualidade em saúde · Quedas de doentes no internamento $\cdot$ Custos hospitalares

\section{Resumo}

Os gastos com a saúde em Portugal aumentaram significativamente nas últimas décadas, apesar de um esforço de contenção desde 2009. As quedas de doentes são episódios frequentes nos internamentos de qualquer Unidade Hospitalar, que podem originar lesões, hospitalizações mais prolongadas, atraso na reabilitação e aumento de custos para o serviço. A presente investigação tem por objetivo analisar quais as causas, consequências e custos de episódios de quedas notificadas com lesão, de doentes internados numa Unidade Hospitalar de Lisboa, com natureza de entidade pública. Utiliza-se uma abordagem quantitativa, do tipo correlacional-preditivo-transversal, através de uma análise retrospetiva de 153 notificações de incidentes de quedas com lesão, durante o ano de 2013. A grande maioria dos doentes tinha idade compreendida entre 71 e 85 anos, sendo a média da idade de 72 anos. 0 principal motivo que levou à queda foi a busca da satisfação das suas necessidades fisiológicas $(47,7 \%)$, com probabilidade de ter sido provocada pelo estado de saúde do doente $(67,3 \%)$, com maior incidência de escoriações e hematomas (em igual número $28,1 \%$ ). Os casos de quedas com um nível de lesão moderado e grave são os que representam um maior valor médio dos custos, sendo as lesões graves de origem ortopédica as que acarretaram maiores custos para a Unidade Hospitalar.

(c) 2018 The Author(s). Published by S. Karger AG, Basel on behalf of NOVA National School of Public Health

\section{Falls in Hospital Stays - Causes, Consequences and Costs: Case Study in a Lisbon Hospital Unit}

\section{Keywords \\ Healthcare economics · Healthcare quality · Patient falls during hospitalization $\cdot$ Hospital costs}

\section{Abstract \\ Health expenditure in Portugal increased significantly in recent decades, despite an effort to cut costs since 2009. Patient falls are frequent episodes throughout the period of stay in any hospital, which can lead to injuries, longer hospitalizations, delays in rehabilitation, and increased}

\section{KARGER}

E-Mail karger@karger.com www.karger.com/pjp

Karger Open access (c) 2018 The Author(s). Published by S. Karger AG, Basel on behalf of NOVA National School of Public Health

This article is licensed under the Creative Commons Attribution NonCommercial-NoDerivatives 4.0 International License (CC BY NC-ND) (http://www.karger.com/Services/OpenAccessLicense) Usage and distribution for commercial purposes as well as any distribution of modified material requires written permission.
Ana Lúcia Romão

CAPP/ISCSP, Centro de Administração e Políticas Públicas Instituto Superior de Ciências Sociais e Políticas, Universidade de Lisboa

Rua Almerindo Lessa, PT-1300-663 Lisboa (Portugal)

E-Mail anaromao@iscsp.ulisboa.pt 
costs to the service. This research aims to analyze causes, consequences, and costs of reported fall events which led to injuries in a public Lisbon hospital. The study is based on a predictive, transversal and cross-sectional approach, through a retrospective analysis of 153 fall incident reports that led to injuries during the year of 2013. The vast majority of patients were between 71 and 85 years old, with an average of 72 years of age. The main reason that led them to fall was autonomous motion to relieve themselves ( $47.7 \%)$, likely caused by patient's health condition (67.3\%), with a higher incidence of bruises and hematomas (both 28.1\%). Cases of falls with moderate and severe levels of injury are those that lead to higher average costs, whereas serious orthopedic injuries lead to higher costs for the hospital.

○ 2018 The Author(s). Published by S. Karger AG, Basel on behalf of NOVA National School of Public Health

\section{Introdução}

Nos países da OCDE, nas últimas décadas do século XX e início do século XXI, o nível de despesa com a saúde aumentou acima do crescimento do PIB [1]. O contexto de crise levou muitos países da Europa a decidir pela redução dos gastos com a saúde como forma de controlar o crescimento da despesa pública, o aumento do défice e o endividamento do país [2].

Nos últimos anos, os gestores hospitalares não se têm poupado a esforços, na tentativa de diminuir a despesa, promovendo medidas de controlo e contenção de custos, bem como o desenvolvimento de novos modelos de gestão com o objetivo de atingirem uma maior eficiência. Tendo sempre presente que "uma política de saúde responsável, em Portugal, não deve gastar menos em saúde, mas gastar melhor" [3].

A segurança do doente tem sido um dos temas com maior destaque nos últimos tempos, tornando-se numa crescente preocupação para as Unidades Hospitalares (UH's), decisores políticos, profissionais de saúde, doentes e seus familiares. Pode-se afirmar que a segurança do doente no internamento surge como o paradigma da qualidade dos cuidados de saúde. A busca pela melhoria dos cuidados de saúde converteu-se numa prioridade máxima, sendo a Segurança do Doente (SD) um instrumento indispensável para a sua garantia.

Um internamento, por mais curto que seja, altera profundamente os hábitos de vida diários do doente que, associado a uma saúde debilitada, aumenta consideravelmente o risco de um evento adverso (EA). Cerca de 10 a $15 \%$ dos doentes internados em UH's sofrem EA's, dos quais 5 a 7\% sofrem lesões graves, podendo mesmo resultar em morte [4].

Um estudo realizado sobre os custos diretos resultantes de EA's na Holanda concluiu que $1 \%$ do orçamento nacional, destinado aos cuidados de saúde em 2004, foi gasto em doentes que sofreram EA's no internamento [5].

Dos diferentes EA's que surgem em internamento, nomeadamente efeitos de fármacos, dispositivos médicos, relacionados com anestesia e cirurgias, infeções associadas aos cuidados de saúde, quedas e lesões por úlceras de pressão, optou-se por estudar o incidente notificado de quedas de doentes no internamento.

Como objetivo geral, pretendemos analisar quais as causas, consequências e custos de episódios de quedas notificadas com lesão, de doentes internados numa Unidade Hospitalar.

\section{Gestão de unidades hospitalares}

As UH's são estruturas de natureza única, onde a unidade de produção é o doente ou caso tratado (episódio) [6]. Cada doente é um produto irrepetível, em que os cuidados recebidos dependem da gravidade da doença, bem como do tratamento, dependendo do médico que o prescreve, entre outros fatores.

As UH's com natureza de entidade pública não se devem reger pela obtenção de lucros mas pelo foco no seu objetivo final: prestar cuidados de excelência a quem a eles recorre. No entanto, devem conhecer bem os seus custos, para os poder gerir de forma eficiente, implementando medidas de controlo, monitorizando e contendo os custos para se tornarem eficazes no seu objetivo principal.

A qualidade dos serviços de saúde deve ser vista como uma questão básica e intrínseca à prestação de cuidados e não somente como uma dimensão desejável, mas dependente [7], ou seja, "fazer a coisa certa para o doente certo, no tempo certo, de maneira adequada de forma a atingir os melhores resultados possíveis" [8].

O sistema de classificação de doentes em Grupos de Diagnóstico Homogéneos (GDH) é apontado como o sistema mais preciso e operacional de classificação de doentes tratados em UH [9].

A cada GDH é associado um peso relativo, isto é, um coeficiente de ponderação que reflete o custo previsto com o tratamento de um doente típico do mesmo grupo, face ao custo médio do doente típico a nível nacional. $\mathrm{O}$ índice de casemix de um hospital resulta do cálculo entre o número de doentes equivalentes ponderados pelos pesos relativos dos respetivos $\mathrm{GDH}$ e o número total de doentes equivalentes [10]. 
Desde 2015 que está em vigor um agrupador GDH do tipo All Patientes Redefined (APR) que implica novos pesos relativos para cada GDH. Esta nova versão apresenta uma vertente clínica mais reforçada e intuitiva para os profissionais de saúde, permitindo efetuar uma caracterização mais detalhada da morbilidade hospitalar, quanto à complexidade dos doentes tratados [11].

\section{Quedas}

A International Quality Indicator Project define queda como qualquer movimento do doente não planeado, caindo para o chão ou de um plano para o outro. Define, ainda, como queda documentada, aquela que é registada no processo clínico do doente, numa descrição do incidente, num relatório do Gabinete de Segurança do Doente (GSD) ou em qualquer outro registo oficial do hospital [12].

As quedas, em meio hospitalar, constituem um risco real e representam um indicador de qualidade [13]. Este indicador tem grande impacto na qualidade dos cuidados de enfermagem e na segurança do doente. Um indicador de qualidade pode ser definido como "a medida utilizada para determinar, através do tempo, o desempenho das funções, processos e resultados de uma instituição" [14].

Um internamento, por mais curto que seja, altera profundamente os hábitos de vida diários do doente, o que associado a uma saúde debilitada, aumenta consideravelmente o risco de queda (RQ). As quedas são consideradas acontecimentos traumáticos e multifatoriais; normalmente são involuntárias e imprevisíveis, podendo o mesmo doente cair mais que uma vez, provocando-lhe com frequência lesões. Estas acarretam consequências para o próprio cuidador e para a sociedade [15].

A queda de um doente internado numa UH é o incidente de segurança que surge com mais frequência, compreendendo um terço de todos os incidentes notificados [16]. Perante uma população envelhecida, com limitações físicas e cognitivas, torna-se um grande desafio evitar este tipo de incidentes.

As quedas são a terceira causa mais comum de morte por lesão não intencional em todas as faixas etárias e a primeira causa entre pessoas com 65 anos ou mais. Porém, cerca de $96 \%$ das quedas não resultaram em qualquer lesão ou resultaram em lesão minor, sendo as incisões, contusões e escoriações as mais relatadas; apenas $1 \%$ resultaram em lesões graves [16].

As lesões graves, na sua maioria ortopédicas, têm um impacto devastador para a qualidade de vida do doente, bem como, a nível económico para as UH's, nomeadamente, custos acrescidos com cirurgias, aumento da du- ração do internamento e, menos comum, processos jurídicos de litígio. Este acréscimo de custos nunca está presente no Contrato-Programa anual. Sendo estes incidentes conotados como uma falha na segurança da UH para com o doente, o Estado nunca financia, tornando-se num custo acrescido.

Impedir a queda de um doente é um desafio porque a sua segurança pode depender da necessidade de ir contra o direito de fazer as suas próprias escolhas, a sua dignidade e privacidade, tais como: levantar da cama, tomar banho, ir ao WC, andar sem a ajuda de terceiros. As quedas de doentes internados não podem ser totalmente evitadas, ocorrendo apesar das medidas preventivas [17].

Alcançar zero quedas não é um objetivo realista. A recuperação do doente numa UH envolve sempre RQ, à exceção dos serviços onde os doentes não sejam capazes de recuperar a sua independência e/ou voltar para casa [16].

Um estudo realizado no Hospital de Braga identificou a queda de doentes como o incidente mais reportado, sendo a sua notificação feita para o GSD, na sua maioria por enfermeiros (95\%). Cerca de 60,1\% dos doentes foram avaliados em relação ao RQ nas primeiras $24 \mathrm{~h}$ de internamento: 32,1\% apresentava RQ elevado, 40,5\% RQ médio e $27,5 \%$ RQ baixo [18].

Um estudo realizado em 2012 concluiu que, tendo por base de referência a classe sem RQ, os doentes classificados com baixo RQ tinham três vezes mais probabilidade de cair e doentes identificados com alto RQ tinham sete vezes essa probabilidade, no mesmo período de internamento [19]. Outros estudos têm também confirmado este tipo de relação [20].

\section{Métodos}

Optou-se por um estudo de abordagem quantitativa, do tipo correlacional-preditivo-transversal, recorrendo ao programa SPSS, versão 22.0 .

A recolha dos dados foi realizada através da análise e interpretação dos processos médicos, de enfermagem e de exames complementares de diagnóstico, tendo em conta o número de episódio de cada doente notificado ao GSD por queda com lesão.

Os custos com exames complementares de diagnóstico e tratamentos foram apurados com base na tabela da Portaria n. ${ }^{\circ}$ 163/2013 [21].

Os GDH dos doentes submetidos a cirurgias foram fornecidos pelo Serviço de GDH - Codificação Clínica da UH.

Em relação aos custos com número de dias de internamento a mais, devido a quedas e outras intervenções, tais como: ajuste de terapêutica, fisioterapia, encaminhamento para unidades de cuidados continuados, não foi possível apurar, uma vez que não existe referência nos processos clínicos dos doentes desse tipo de intervenções associadas ao incidente de queda. 
Table 1. Estatísticas da idade em função do género

\begin{tabular}{lllllll}
\hline & Mínimo & Máximo & Mediana & Média & Desvio padrão & Moda \\
\hline Masculino $(n=97)$ & 32 & 90 & 73 & 70,93 & 12,60 & 81 \\
Feminino $(n=56)$ & 34 & 93 & 77,5 & 74,36 & 13,37 & 81 \\
\hline Total da amostra $(n=153)$ & 32 & 93 & 76 & 72,18 & 42,95 & 81 \\
\hline
\end{tabular}

A população alvo corresponde ao número total de notificações de quedas no ano de 2013 na UH, que equivale a 894 notificações. A UH abrange 15 áreas médicas diferentes, com um total de 1.278 camas, com uma taxa de ocupação de $91,76 \%$.

A população acessível engloba o número total de notificações de quedas em 2013 na UH, em duas grandes áreas, Medicina e Cirurgia, onde se notificaram 580 quedas. A Área da Medicina tem uma lotação de 394 camas, com uma taxa de ocupação de $89,84 \%$ diária. Por sua vez, a Área da Cirurgia tem uma lotação de 244 camas, com uma taxa de ocupação de $87,45 \%$ diária. No total corresponde a 23.814 doentes, podendo afirmar-se que cerca de $2,2 \%$ destes doentes sofreram uma queda acidental.

Para reduzir o erro de amostragem utilizou-se o método não probabilístico por escolha racional ou típica, sendo a amostra definida através de um traço caraterístico, o estudo de quedas apenas com lesão. A amostra é constituída por 153 notificações de quedas de doentes com lesão.

Numa primeira fase da análise, a estatística descritiva permitiu estudar as variáveis em estudo, com a análise exploratória das variáveis contínuas e a análise de frequências das variáveis categóricas.

A segunda parte da análise correspondeu ao estudo inferencial dos dados, no sentido de validar ou rejeitar as hipóteses de investigação formuladas. Numa fase prévia à verificação das hipóteses foi testada a normalidade das variáveis para validar a utilização dos testes paramétricos. Para tal utilizou-se o teste de KolmogorovSmirnov cuja hipótese nula estipula que as variáveis seguem uma distribuição normal, para um intervalo de confiança de $95 \%$.

Apenas a variável hora da queda revelou ter uma distribuição normal $(p>0,05)$ e o teste de Levene permitiu confirmar a hipótese da igualdade das variâncias $(p>0,05)$, validando a utilização do teste paramétrico de Análise de Variância (ANOVA) para comparar os valores médios da hora da queda em função do nível de gravidade da lesão. A hipótese nula do teste ANOVA estipula a igualdade das médias entre os grupos para um intervalo de confiança de $95 \%$. Assim, quando o valor de $p$ observado é inferior a 0,05 conclui-se sobre a existência de diferenças estatisticamente significativas [22].

Nas análises que incluíram as restantes variáveis foi utilizado o teste não paramétrico de independência do Qui-quadrado de Pearson cuja hipótese nula considera que as variáveis em análise são independentes, para um intervalo de confiança de 95\%.

Ao nível dos custos associados a atos clínicos pretendeu-se avaliar a relação estatística entre os valores médios dos custos tipificados e as variáveis nível de gravidade e Monitorização e Avaliação do Risco de Queda (MARQ). A hipótese nula do teste de Kruskal-Wallis estipula a igualdade das médias entre os grupos para um intervalo de confiança de $95 \%$, pelo que se o valor de $p$ for inferior a 0,05 temos de concluir a favor da hipótese alternativa de que os valores médios diferem entre os grupos em análise.
A correlação estatística entre as variáveis numéricas, nomeadamente entre os custos e a idade dos doentes, por um lado, e entre os custos e a hora da queda, por outro, foi avaliada através do Teste de Correlação de Pearson. O coeficiente de correlação dos dados mede a direção da força de cada relação linear entre as duas variáveis.

\section{Análise e Discussão dos Resultados}

A análise descritiva dos dados empíricos permitiu observar que os doentes com quedas notificadas se concentravam entre os 71 e os 85 anos, o que confirma as afirmações dos autores que abordam a variável idade, segundo os quais a maioria das quedas surge acima dos 65 anos (Tabela 1).

Quando se compara a média das idades em função do nível de gravidade da lesão não foram observadas diferenças significativas, porém conseguiu-se observar que a média das idades foi superior nos doentes com nível de lesão moderado (86) e nos doentes com um nível de lesão grave (81). Esta observação pode dever-se à vulnerabilidade do doente face à lesão e ao efeito da doença coexistente, uma vez que, quanto mais idoso, mais comorbilidades associadas [16].

Em relação à frequência temporal dos episódios de queda, optou-se por realizar uma comparação entre a população acessível e a amostra, melhorando a validação da análise e favorecendo uma tomada de decisão mais fundamentada, numa possível implantação de novas medidas preventivas pela $\mathrm{UH}$.

Ao nível dos doentes com e sem lesão $(n=580)$ os meses em que foram registados um maior número de quedas foram os meses de abril $(n=61 ; 10,5 \%)$, dezembro $(n=$ $56 ; 9,7 \%)$, fevereiro $(n=55 ; 9,5 \%)$ e janeiro $(n=54 ; 9,3 \%)$. Encontraram-se diferenças significativas em comparação com os meses da amostra $(n=153)$, em que foram registadas mais quedas no mês de outubro $(n=21 ; 13,7 \%)$, seguindo-se os meses de agosto $(n=16 ; 10,5 \%)$, março e julho com 15 quedas cada $(9,8 \%)$. 


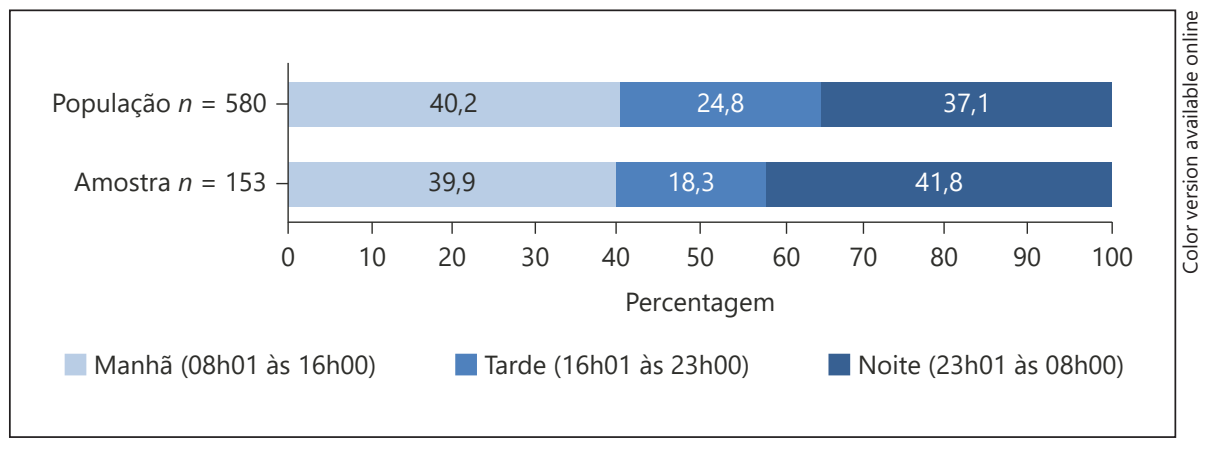

Fig. 1. Quedas notificadas por período.

O dia da semana em que se registou uma maior ocorrência de quedas foi à terça-feira $(20.3 \%$ dos casos da amostra e $15,7 \%$ dos casos da população acessível). Seguindo-se o fim-de-semana: para a amostra observaram-se mais episódios de queda ao domingo $17 \%(n=26)$, enquanto para a população acessível foi ao sábado $14,3 \%$ $(n=83)$.

Na população acessível foi no período da manhã onde se registou um maior número de quedas $(n=233 ; 40,2 \%)$, seguindo-se o período da noite $(n=215 ; 37,1 \%)$ e apenas $24,8 \%(n=144)$ no período da tarde. Na amostra o período onde ocorreram um maior número de quedas com lesão foi à noite $(n=64 ; 41,8 \%)$, seguindo-se o período da manhã $(n=61 ; 39,9 \%)$ e apenas $18,3 \%(n=28)$ no período da tarde (Fig. 1).

A hora em que foi notificado um maior número de quedas foi às $10 \mathrm{~h}$, pelo facto de ser neste momento do dia que os doentes fazem a sua higiene pessoal. Seguindo-se às $24 \mathrm{~h}$ e $6 \mathrm{~h}$, aqui talvez pela ineficaz iluminação e maior sonolência com que se encontram quando se levantam a meio da noite por necessidades fisiológicas.

No final dos três turnos (manhã-14 h, tarde-21 h e noite- 6 h) registaram-se outros picos com ocorrência de quedas mais notória na população acessível. Neste horário os enfermeiros e os auxiliares de ação médica encontram-se na enfermaria a atender e a observar os doentes de forma mais individual, limitando a sua disponibilidade imediata para auxiliar outros doentes. Esta indisponibilidade leva a que alguns doentes tentem atender às suas necessidades sozinhos originando possíveis episódios de queda.

Verificou-se que no geral das quedas notificadas, estas corresponderam a uma queda por doente num mesmo internamento ( $n=148 ; 96,7 \%)$, sendo que apenas foram observados dois doentes com duas quedas, outros dois doentes que registaram três quedas e apenas um doente que registou quatro quedas.

Quedas em internamento hospitalar: causas, consequências e custos
Verificou-se que 85,6\% do número de episódios de quedas notificados tinham sido previamente avaliados, segundo a Escala de Morse. Dos quais 37,9\% de risco médio prévio à queda e $32,7 \%$ com alto risco de queda, valores semelhantes a outros estudos [18].

Observou-se ainda que, na maioria dos casos das quedas com lesão, os doentes se encontravam orientados (77\%), não agitados (95\%) e sem medidas de contensão mecânica (93\%). Na maioria dos casos (79\%), as quedas dos doentes em internamento não foram observadas ou presenciadas. Verificou-se uma associação estatística significativa entre a inexistência de grades elevadas e contensão mecânica e o estado de orientação do doente, sobretudo em virtude do estado de orientação do doente.

O serviço que teve maior número de quedas notificadas foi a medicina $(65,4 \% ; n=100)$, seguindo-se a cirurgia $(17,6 \% ; n=27)$, a urologia e o serviço de doenças infectocontagiosas com $(3,9 \% ; n=6)$, o de nefrologia $(3,3 \%$; $n=5)$, o de gastroenterologia $(2,6 \% ; n=4)$, o de pneumo$\operatorname{logia}(2 \% ; n=3)$ e, por fim, o de dermatologia $(1,3 \% ; n=2)$.

$\mathrm{O}$ local mais frequente das quedas dos doentes em internamento foi o quarto $(62,1 \% ; n=95)$, seguindo-se as instalações sanitárias $(19,6 \% ; n=30)$. No corredor foram notificadas $8,5 \%(n=13)$ das quedas, no duche $7,2 \%(n=$ $11)$, na rua $2 \%(n=3)$ e no refeitório uma queda $(0,7 \%)$.

O motivo do levante mais frequente foram as necessidades fisiológicas $(47,7 \% ; n=73)$, seguindo-se a confusão do doente $(15 \% ; n=23)$ e a higiene $(14,4 \% ; n=22)$. As necessidades fisiológicas dos doentes têm-se associado, com alguma frequência, ao nível de gravidade da lesão grave [16].

O motivo mais frequente das quedas foi o estado de saúde do doente $(67,3 \% ; n=103)$, seguindo-se os fatores ambientais $(21,6 \% ; n=33)$, tais como: água e/ou urina no chão, piso degradado, falta de travões nos cadeirões, camas e macas, a resposta ao tratamento, medicação ou anestesia $(7,8 \% ; n=12)$. 
Fig. 2. Tipo de lesão.

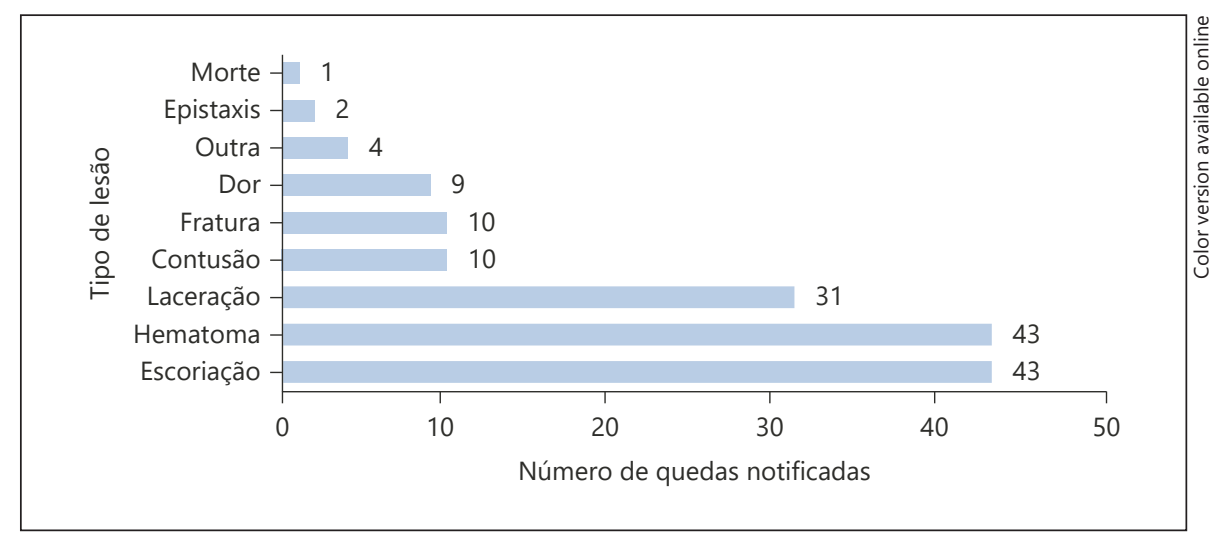

Table 2. Tipologia de custos

\begin{tabular}{lc}
\hline Tipologia de custos & Valor, EUR \\
\hline Cirurgia & 28.257 \\
Observações médicas & 6.744 \\
Tratamentos & 3.756 \\
Tomografias computorizadas & 3.720 \\
Transporte & 2.400 \\
Raio-X & 439 \\
Ressonância magnética & 256 \\
Outros exames & 457 \\
Outros custos & 4.613 \\
\hline Total & 50.641 \\
\hline
\end{tabular}

O estado de saúde, que se manifesta na debilidade de saúde individual de cada doente, foi o motivo que surgiu com mais frequência, porém quando associado estatisticamente com o nível de gravidade da lesão não revelou uma associação estatística significativa. $\mathrm{O}$ doente idoso quando é internado numa $\mathrm{UH}$, por mais curto que seja o internamento, modifica profundamente os hábitos de vida diários, o que associado a um estado de saúde debilitado, aumenta consideravelmente o RQ [15].

$\mathrm{Na}$ maioria dos casos das quedas notificados, o nível de gravidade da lesão foi ligeiro $(79,1 \% ; n=121)$, em 22 casos $(14,4 \%)$ foi moderado e em apenas dez casos se observou um nível de gravidade grave (6,5\%); que geraram diferentes tipos de lesão (Fig. 2).

Dos dez casos de quedas notificados com nível de gravidade grave, dois apresentaram traumatismos crânioencefálicos (hematomas subdural grave, um levou a morte o outro reverteu), um traumatismo do braço (fratura sem necessidade de cirurgia - tratamento conservador) e os restantes sete sofreram fraturas da perna, tendo sido apenas seis operados.

O nível de gravidade da lesão e o tipo de queda não confirmam associação estatística significativa, contudo o nível de gravidade da lesão e o tipo de lesão apresentam uma associação estatística altamente significativa.

De todas as notificações de quedas, 93 casos $(60,8 \%)$ foram observados por urgência interna. Na sequência da lesão, houve necessidade de 22 casos (14\%) serem observados por outra especialidade, das quais se destacaram onze observações por ortopedia $(7,2 \%)$, cinco por neurocirurgia $(3,3 \%)$, dois por neurologia e dois por maxilo-facial $(1,3 \%)$.

De todos os episódios de quedas notificados, sete foram submetidos a cirurgia; quatro foram submetidos a redução fechada de fratura do fémur com fixação interna; um a redução aberta de fratura de fémur, com fixação interna; um a desbridamento e sutura da ferida escrotal (menos complexa) e outro a substituição parcial da anca e vertebroplastia percutânea.

Quando se realiza a associação estatística entre o nível de gravidade da lesão e o tipo de cirurgia resulta numa associação estatística altamente significativa, uma vez que, os atos cirúrgicos são extremamente invasivos para o doente, tornando-o mais vulnerável e debilitado. Logo, se se corre o risco de submeter o doente a uma cirurgia o nível de gravidade da lesão tem que ser grave. Foram precisamente os custos com as cirurgias que implicaram mais gastos na $\mathrm{UH}$.

No ano de 2013, as quedas com lesão representaram um total de custos de EUR 50.641, o que corresponde, em média, a EUR 331 por cada queda notificada $(n=153)$. Apesar de apenas terem sido registados 10 casos de GDH cirúrgico, esta foi a rubrica onde o volume de custos foi superior (Tabela 2). 
Table 3. Síntese dos custos totais médios da população acessível

\begin{tabular}{lccc}
\hline Nível de gravidade & $\begin{array}{l}\text { Custo médio por episódio } \\
\text { de queda, EUR }\end{array}$ & $\begin{array}{l}\text { Número total de } \\
\text { episódios de queda }\end{array}$ & $\begin{array}{l}\text { Custos totais, } \\
\text { EUR }\end{array}$ \\
\hline Sem lesão & 46 & 427 & 19.642 \\
Lesão ligeira & 117 & 121 & 14.185 \\
Lesão moderada & 296 & 22 & 6.504 \\
Lesão grave & 2.995 & 10 & 29.952 \\
\cline { 2 - 3 } & & Total & 70.283 \\
\hline
\end{tabular}

Realizou-se uma estimativa para os custos com notificações de quedas sem lesão, através da contabilização do total de custos com a observação pela urgência médica nos turnos da tarde e noite, o valor encontrado foi dividido pelo total de notificações de quedas sem lesão e somou-se a esse valor uma média de custo de exames de EUR 10, perfazendo um total de EUR 46; totalizando EUR 19.642 (Tabela 3).

\section{Conclusões}

Com o aumento da esperança média de vida, os consumidores dos cuidados de saúde hospitalares são cada vez mais idosos, apresentando múltiplas comorbilidades. Daí se verificar uma grande concentração de quedas notificadas de doentes com idade compreendidas entre os 71 e os 85 anos.

$\mathrm{Na}$ tentativa de dar resposta a esta realidade, as $\mathrm{UH}$ têm aumentado o número de camas de medicina, numa tentativa de uma melhor gestão de recursos, por isso a grande discrepância no número de notificações de quedas por serviço.

A maioria das quedas ocorre quando o doente se encontra sozinho, na ausência de profissionais de saúde que o possam acompanhar, cuidar e vigiar.

O principal motivo que levou o doente a cair foi a busca da satisfação das suas necessidades fisiológicas, seguindo-se por períodos de confusão e a realização da própria higiene. O motivo com mais probabilidade de ter provocado a queda foi o estado de saúde do doente, seguindo-se os fatores ambientais e a resposta ao tratamento, medicação ou anestesia.

As lesões que surgiram com mais frequência após o episódio de queda foram escoriações e hematomas, lacerações e fraturas.
Analisou-se a média de custos (por tipologia) em função da MARQ com resultado de alto risco, não se verificando a existência de diferenças significativas.

Quando se compara a média de custos em função do nível de gravidade da lesão concluiu-se que os valores diferem em termos do nível de gravidade da lesão. Em geral, os custos diretos tendem a aumentar com a severidade da lesão, sendo as quedas com um nível de lesão moderado e grave as que representam um maior valor médio dos custos.

Em termos de custos com os episódios de queda com lesão, concluiu-se que foram as lesões graves de origem ortopédica que maiores custos acarretaram, seguidas pelos custos com as observações médicas e os custos com os tratamentos.

No ano de 2013, para a população acessível desta UH, o total de custos com incidentes de quedas notificadas com lesão apontam para um total de EUR 50.641 e sem lesão para um total de EUR 19.642. Logo, estima-se que a $\mathrm{UH}$ teve um total de custos com episódios de queda aproximado de EUR 70.283. Caso extrapolássemos estes valores para a população alvo desta $\mathrm{UH}$, os custos ascenderiam a EUR 108.333.

Os dados apontam que é sobretudo o nível de gravidade da lesão que tem relação estatística com os custos hospitalares das quedas dos doentes durante internamento.

Os custos com medicação, dias de internamento e/ou danos de índole psicológica ou social/económica, não foram possíveis de quantificar, uma vez que, estes não são relatados em todo o processo clínico.

\section{Conflito de interesses}

As autoras declaram não haver conflito de interesses. 


\section{References}

1 OECD: Value for Money in Health Spending. Paris, OECD Publishing, 2010.

2 Mladovsky P, Srivastava D, Cylus J, Karanikolos M, Evetovits T, Thomson S, et al: Health policy in the financial crisis. Eurohealth 2012; 18:3-6.

3 Campos AC: Reformas da saúde: o fio condutor. Coimbra, Edições Almedina, 2008.

4 Uva AS, Serranheira F: A segurança do doente também depende da saúde e segurança de quem presta cuidados. Rev Hospital Futuro 2008;10:1-2.

5 Hoonhout L, Bruijne M, Wagner C, Zegers M, Waaijman R, Spreeuwenber, et al: Direct medical costs of adverse events in Dutch hospitals. BMC Health Serv Res 2009;9:27. https://www.biomedcentral.com/14726963/9/27.

6 Reis V: Gestão em saúde: um espaço de diferença. Lisboa, ENSP, 2007.

7 Delgado M: A melhoria contínua da qualidade; in Borges M, Campos L, Portugal R (eds): Governação dos hospitais. Alfragide, Casa das Letras, 2009, pp 45-55.

8 Silva M: Gestão da qualidade em cuidados de saúde. Lisboa, Monitor - Projetos e Edições, 2013.
9 Simões J: Retrato político da saúde: dependência do percurso e inovação em saúde: da ideologia ao desempenho. Coimbra, Almedina, 2004.

10 Ministério da Saúde: Portal da Codificação Clínica e dos GDH. Grupos de Diagnósticos Homogéneos (GDH). Lisboa, Ministério da Saúde, 2014. http://portalcodgdh.min-saude. pt/index.php/Grupos_de_Diagn\% C3\%B3sticos_Homog\%C3\%A9neos_(GDH) (acedido a 3 de agosto de 2014).

11 Portaria n. ${ }^{\circ} 234 / 2015$. Diário da República n. ${ }^{\circ}$ 153/2015. Série I. (2015-08-07) 5516-654. Regulamento e Tabelas de Preços das Instituições e Serviços Integrados no Serviço Nacional de Saúde.

12 International Quality Indicator Project. Indicator 13: Falls. Newcastle, University of Newcastle, 2005.

13 Saraiva D, Louro I, Ferreira L, Batista P, Pina S, Ramalhinho J: Quedas: indicador de qualidade assistencial. Nursing 2008;18:28-35.

14 Joint Commission International: Padrões de acreditação da Joint Commission Internacional para Hospitais, ed 3. Rio de Janeiro, Consórcio Brasileiro de Acreditação, 2008.
15 Almeida R, Abreu C, Mendes A: Quedas em doentes hospitalizados: contributos para uma prática baseada na prevenção. Rev Enferm Ref 2010;3:163-172.

16 Healey F, Scobie S: Slips, Trips and Falls in Hospital: The Third Report from the Patient Observatory. London, National Patient Safety Agency, 2007.

17 Nadkarni J, Iyengar K, Dussa C, Watve S, Vishwanath K: Orthopaedic injuries following falls by hospital in-patients. Gerontology 2005;51:329-333.

18 Cunha S: Quedas dos doentes no Hospital de Braga: análise dos eventos e custos associados; Dissertação de Mestrado; Braga, 2013.

19 Costa-Dias M, Martins T, Araújo F: Estudo do ponto de corte da Escala de Quedas de Morse (MFS). Rev Enferm Ref 2014;4:65-74.

20 McAlister S: APR DRG weights and the relationship to patient falls. Nurs Econ 2009;27: 119-123.

21 Portaria n. ${ }^{\circ}$ 163/2013. Diário da República n. ${ }^{\circ}$ 80/2013. Série I. (2013-04-24) 2495-606. Tabelas de Preços e Regulamento a praticar pelo Serviço Nacional de Saúde.

22 Pestana ML, Gageiro JN: Análise de dados para ciências sociais: a complementaridade do SPSS, ed 6. Lisboa, Edições Sílabo, 2014. 